\title{
An unusual cause of eosinophilic pleural effusion and migrating biliary stenosis: Strongyloides stercoralis infection in a young immunocompetent man
}

\author{
Elisa Paolucci, ${ }^{1}$ Eva Tonveronachi, ${ }^{1}$ Chiara Praticò, ${ }^{2}$ Maria Cristina Zani, ${ }^{1}$ Maria Cristina Paonessa, ${ }^{1}$ \\ Michele Maria Domenico Imperatore, ${ }^{1}$ Beniamino Praticò ${ }^{1}$ \\ ${ }^{1}$ Internal Medicine Unit, Bufalini Hospital, Cesena (FC); ${ }^{2}$ Medicina Ospedale S. Orsola-Malpighi, Bologna, Italy
}

\begin{abstract}
We present the case of a 33-year-old Italian man who came to our attention for epigastralgia associated with polyserositis (pleural, pericardial, and peritoneal effusion with the involvement of the Douglas excavation), in the absence of a significant medical history. Laboratory analysis revealed exudative eosinophilic pleural effusion; serial imaging techniques showed a transient stenosis of the right hepatic duct and a subsequent stenosis of the left hepatic duct. After several negative serological investigations, a positive anti-strongyloides immunoglobulin $\mathrm{G}$ antibodies titer rose suspicions of Strongyloides infection, which was confirmed by positive stool sample for parasite. Ivermectin-therapy was started and the patient has fully recovered.
\end{abstract}

\section{Introduction}

Strongyloides is a parasite that lives in the soil of rural areas of tropical and subtropical regions and occurs sporadically in temperate areas. ${ }^{1}$ Literature reports several cases of Strongyloides infection in immunosuppressed patients predominantly, with malignancies (especially hematological), autoimmune history, malnutrition, or with a history of alcohol abuse and cirrhosis or of travels in endemic countries. ${ }^{2}$

A prompt treatment with ivermectin can lead to a rapid resolution of a potentially lethal infection. ${ }^{3}$

Correspondence: Elisa Paolucci, Internal Medicine Unit, Bufalini Hospital, via Ghirotti 256, Cesena (FC), Italy. Tel.: +39.0547.352875.

E-mail: elisa.paolucci@auslromagna.it

Key words: Pleural effusion; eosinophilia; hepatic duct stenosis; Strongyloides stercoralis; ivermectin.

Conflict of interests: the authors declare no potential conflict of interests.

Received for publication: 5 December 2018.

Revision received: 31 January 2019.

Accepted for publication: 31 January 2019.

This work is licensed under a Creative Commons Attribution NonCommercial 4.0 License (CC BY-NC 4.0).

(C) Copyright E. Paolucci et al., 2019

Licensee PAGEPress, Italy

Italian Journal of Medicine 2019; 13:116-120

doi:10.4081/itjm.2019.1121

\section{Case Report}

A 33-year-old man, with modest smoking habit (1 $\mathrm{pk} / \mathrm{y}$ ), works in a waste disposal site (sewage sludge), lives in the countryside, owns hens, and consumes field herbs and rarely raw crustaceans. No significant past medical history is reported, except for a right knee arthroscopy in 1998. He has a family history of diabetes, and he does not take any medicine.

Before coming to our attention, he underwent some diagnostic test for a persistent diarrhea. Hematological screening for celiac disease, abdominal ultrasound and colonoscopy resulted negative. His medical history started with an acute episode of sinusitis successfully treated with non-steroidal antiinflammatory drugs, and shortly after he went to the Emergency Room (ER) for an acute episode of epigastralgia, where he was discharged under omeprazole for a suspected gastritis.

About one week later he reported a relapse of epigastralgia, irradiating to the right hypochondrium, associated with nausea. He went to the ER again: the physical examination showed signs of peritonism, while the bedside ultrasound evaluation revealed bilateral medium-basal pleural effusion, with bilateral pulmonary thickening, air bronchogram, circumferential pericardial effusion $>1 \mathrm{~cm}$, mild liquid flap in the Douglas and thickened gallbladder wall. The patient was then admitted to our Department of Internal Medicine.

Upon presentation to the ward the patient appeared alert and orientated, apyretic and eupneic, with normal vital signs. Laboratory tests showed normal white blood cells count with eosinophilia, raised levels of 
inflammatory markers, transaminases and cholestatic indexes (Table 1).

Chest X-ray performed on the first day of hospitalization showed bilateral obliterations of costophrenic angles in the absence of signs of focal parenchymal inflammation; thoracic ultrasound confirmed bilateral pleural effusion. Abdominal ultrasound showed mild hepatomegaly with homogeneous structure, with no focal lesions, thickened gallbladder walls containing sludge and micro calculi, mild splenomegaly with patent and normal splenoportal axis, regular suprahepatic veins, and a moderate peritoneal effusion.

On day 3 the patient underwent both diagnostic and evacuative thoracentesis, with extraction of $1.050 \mathrm{cc}$ of pale yellow pleural fluid with features of exudate according to Light's criteria $[\mathrm{pH} 7.436$, lactate dehydrogenate $(\mathrm{LDH}) 228 \mathrm{U} / \mathrm{L}$; LDH pleura/serum= 0.97]. Cytological examination revealed inflammatory pleural fluid rich in eosinophil granulocytes.

In order to rule out infectious or autoimmune hepatopathy, multiple serological tests were performed and they all turned out to be in the normal range. HIV, cytomegalovirus, Parvovirus B19, hepatitis A, B, C,

Table 1. Baseline laboratory investigation.

\begin{tabular}{lccc}
\hline Parameter & $\mathbf{1}^{\text {st }}$ value & $\begin{array}{c}\mathbf{2}^{\text {nd }} \text { value } \\
\text { (1 month later) }\end{array}$ & $\begin{array}{c}\text { Reference } \\
\text { range }\end{array}$ \\
\hline Hemoglobin (g/dL) & 15.3 & 15.2 & $13.5-17.0$ \\
\hline Red blood cells $\left(\times 10^{12} / \mathrm{L}\right)$ & 5.18 & 10.70 & $4500-5700$ \\
\hline HCT (\%) & 44.7 & 44.1 & $40.0-52.0$ \\
\hline MCV (fL) & 86.3 & 83.9 & $80.0-95.0$ \\
\hline MCHC (g/dL) & 34.2 & 33.3 & $32.0-36.0$ \\
\hline PLT (×10/L) & 388 & 398 & $140-400$ \\
\hline White cell count (×109/L) & 8.48 & 10.05 & $4000-10,000$ \\
\hline Neutrophils (\%) & 54.7 & 55.1 & $40-70$ \\
\hline Lymphocytes (\%) & 23.1 & 27.2 & $20-45$ \\
\hline Monocytes (\%) & 0.81 & 10.4 & $3-10$ \\
\hline Basophils (\%) & 0.8 & 0.5 & $0-1.5$ \\
\hline Eosinophils (\%) & 11.8 & 12.8 & $0-6$ \\
\hline CRP (mg/L) & 20.4 & 2.89 & $<5$ \\
\hline AST (U/L) & 390 & 22 & $<41$ male \\
\hline ALT (U/L) & 595 & 25 & $<41$ male \\
\hline Alkaline phosphatase U/L & 455 & 172 & $40-129$ male \\
\hline GGT U/L & & 124 & $8-61$ male \\
\hline Total bilirubin (mg/dL) & 1.08 & - & $<1.20$ \\
\hline Direct bilirubin (mg/dL) & 0.53 & - & $<0.30$ \\
\hline & & & over 17 \\
\hline
\end{tabular}

$\mathrm{HCT}$, hematocrit; MCV, mean corpuscular volume; $\mathrm{MCHC}$, mean corpuscular hemoglobin concentration; PLT, platelets; CRP, C-reactive protein; AST, aspartate transaminase; ALT, alanine transaminase; GGT, gamma-glutamyltransferase. and E, Herpes Simplex Virus, Epstein Barr Virus, Toxoplasma, Leptospirosis, Mycoplasma pneumoniae, Adenovirus, Enterovirus, Borrelia burgdorferi, Bordetella, Rickettsia, Echinococcus, Amoebiasis, Toxocara, Anisakis, Quantiferon, and stool sample for parasites were tested and proved negative for active infection. Immunology tests like lymphocyte typing, dosage of TSH, LDH, $\beta 2$ microglobulina, ANA, ASMA, LKM, ENA, antiDNA, FR, MPO ANCA, PR3 ANCA, serum trypsin, RAST tests, resulted in the normal range.

We completed the investigations with a computed tomographic (CT) scan. The CT confirmed bilateral pleural effusion, in the absence of parenchymal nodular formations or mediastinal adenopathies, with minimal pericardial effusion. It also revealed hypodense hilar hepatic tissue of $2 \mathrm{~mm}$ associated with perihepatic fluid and hilar, mesenteric and lombo aortic enlarged adenopathies.

Therefore, since the cholangitic pattern was the most important, a magnetic resonance (MR) cholangiography (Figure 1) was performed and showed a stenosis of the right hepatic duct extended for $12 \mathrm{~mm}$, likely due to the extrinsic compression of a kind of solid tissue, with no dilatation of the biliary ducts, and a distended gallbladder with minimal wall thickening, consistent with acute cholecystitis.

In order to rule out biliary tract tumors, a positron-emission tomography scan (Figure 2) was performed. It showed high uptake of the radioactive tracer in the hepatic hilum and in the peripancreatic fluid (SUV 7.23).

An empiric treatment with ciprofloxacin plus ursodeoxycholic acid was started, with a partial

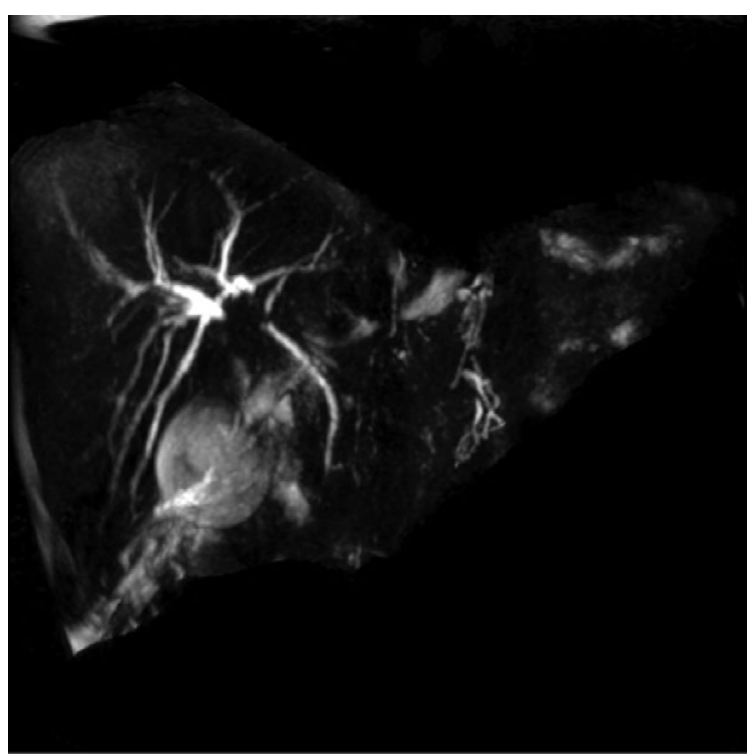

Figure 1. The subsequent magnetic resonance cholangiography showed a stenosis of the right hepatic duct. 
improvement of the inflammatory markers, transaminases and cholestatic indexes. Considering the clinical improvements, we decided to delay the execution of any further investigations, such as percutaneous cholangiography (because of the high pancreatitis risk and, at the same time, because of the absence of a biliary tract dilatation) endobiliary brush or liver biopsy, after a one-month MRI re-evaluation. The patient was discharged with the diagnosis of polyserositis in a cholestatic hepatitis causing the inflammation of the main biliary duct.

The patient carried on with examinations in the outpatient clinic. Laboratory tests showed a raise in eosinophilia and a progressive reduction of hepatic cytolysis indexes (Table 1). On the other side the patient presented a progressive increase in dyspnea. Chest Xray showed a gradual increase in pleural effusion especially in the right lung (Figure 3). Abdominal MRI was repeated one month after the previous one: at this time the right hepatic duct appeared patent while the left duct was inflamed and stenotic with an upward dilatation, and gallbladder showed minimal wall thickening. It also detected a lesion $(25 \times 11 \mathrm{~mm})$ in the hepatic hilum at the emergence of the left hepatic duct, with tumor-like appearance and possibly consistent with an inflammatory pseudotumor or a peripheral cholangiocarcinoma. Moreover, there was abundant abdominal and pleural effusion and enlarged mesenteric lymph nodes.

After 8 days from the MRI, abdominal ultrasound could not detect the hepatic hilar lesion anymore, but showed a persisting inflammatory aspect of the main biliary duct.

Considering the raise in eosinophilia at the blood tests, the persistence of blood and radiological signs of inflammation, we repeated tests for parasites and bacteria, this time including the serology for Strongyloides stercoralis, which turned out positive (anti-strongyloides IgG antibodies). Stool sample confirmed the presence of S. stercoralis larvae.

The patient underwent a five-day antibiotic course of ivermectin per os. Nevertheless, he was still experiencing dyspnea for moderate efforts so that a chest X-ray was repeated and reported persistence of bilateral pleural effusion. Another evacuative thoracentesis was repeated and the patient gradually improved.

A further radiogram performed three months after the treatment showed a complete resolution of the pleural effusion. At this time also serologies and stool examinations for Strongyloides resulted negative.

Since then the patient has been experiencing good clinical conditions.

We concluded that he probably ran into the aforementioned nematode because of his job in a waste disposal site and also because of the onychophagia he suffered from.

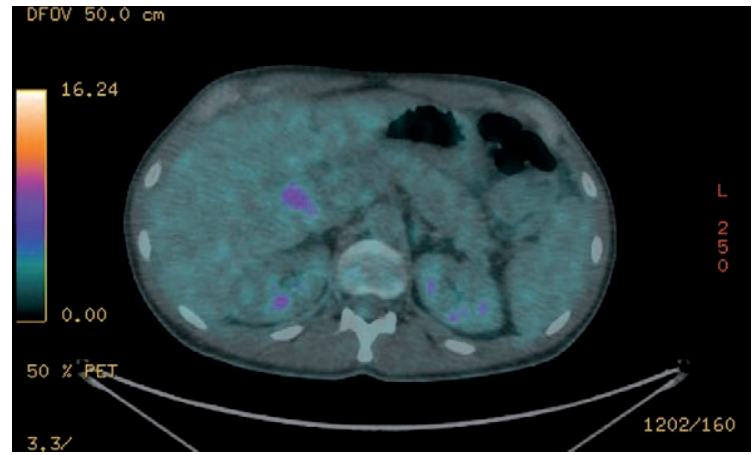

Figure 2. Positron-emission tomographic scan showed high uptake of the radioactive tracer in the hepatic hilum and in the peripancreatic liquid (SUV 7.23).
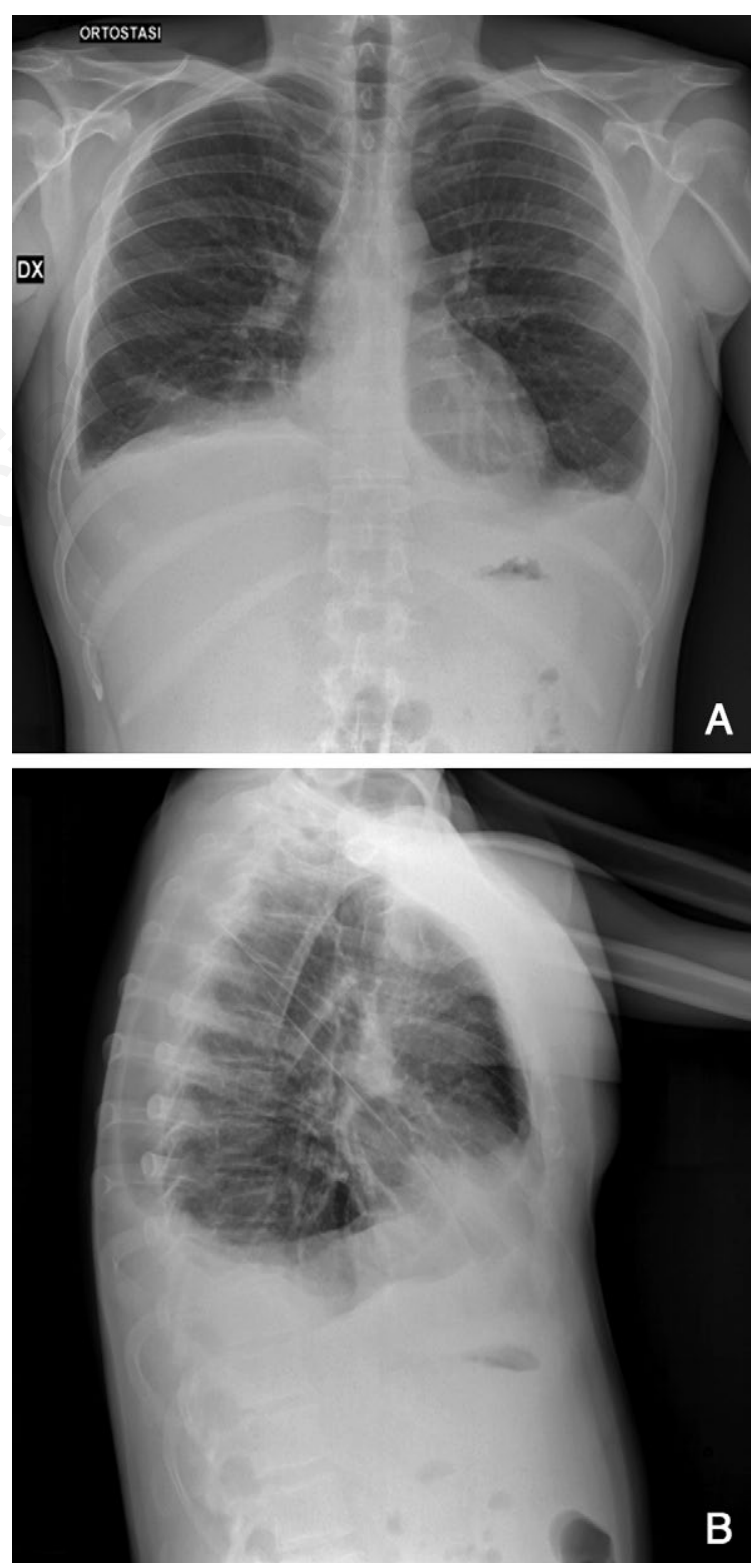

Figure 3. Chest X-ray showed a gradual increase in pleural effusion especially in the right lung: A) frontal view; B) lateral view. 


\section{Discussion and Conclusions}

It is estimated that about 100 million people worldwide have been infected by strongyloidiasis. ${ }^{4}$ Strongyloidiasis is endemic in rural areas of tropical and subtropical countries. ${ }^{5}$ In the past, the infection has also been reported in countries with temperate climate, like Italy.

Larvae live in moist soil and can infect humans by skin contact. ${ }^{6}$ Unlike other helminthic parasites, Strongyloides can complete its life cycle entirely within the human body. The human cycle involves larvae that penetrate the skin, spread into the bloodstream and reach the right heart and then the lungs, where they penetrate into alveolar air sacs. The larvae then ascend bronchi, trachea and larynx and are swallowed. In the small intestine (duodenum and jejunum) they become adult worms, which produce eggs becoming rhabditiform larvae (smaller non-infectious larvae). The rhabditiform larvae mature into filariform larvae that can penetrate the perianal skin and give a start to an autoinfection, with the risk of disseminated disease especially in immunosuppressed patients (e.g., congenital and acquired immunodeficiency as HTLV1 and HIV infections, underlying malignancy, malnutrition, alcoholism, hematopoietic stem cell transplantation, chronic use of steroids or cytotoxic drugs, etc.). ${ }^{7}$ The massive dissemination of larvae to lungs, liver and other organs may induce severe inflammation with symptomatic organ dysfunctions, as in the case of our patient, and even septic shock. ${ }^{8}$

Finally, if defecation takes place outdoors, the feces of the host can infect the soil and spread the infection.

Most of the infected patients have no prominent symptoms. If present, the most common gastrointestinal manifestations include abdominal pain, diarrhea, and involuntary weight loss with malabsorption signs, nausea and vomiting. 9 Pulmonary manifestations include dry cough, throat irritation, wheezing and hemoptysis. Some patients with chronic strongyloidiasis can present some kind of recurrent bacterial pneumonia or can develop asthma that characteristically worsens with corticosteroid use. ${ }^{10}$ There could be a cutaneous reaction where larvae penetrate the skin, urticarial areas near to the anus, but also migrating skin rashes (raised and transient pink lesions related to the dermal migration of larvae, the so called running larvae), with pruritus. ${ }^{11}$

The detection of rhabditiform larvae in stool samples or through serological tests usually makes the diagnosis of uncomplicated strongyloidiasis. However, stool examination is often insensitive for detecting Strongyloides because larvae are excreted only intermittently and this might explain the delay in detecting larvae in the stool sample of our patient. In disseminated strongyloidiasis, larvae can be found also in sputum, bronchoalveolar lavage fluid, and pleural, peritoneal and surgical drainage fluid. ${ }^{12}$ Polymerase chain reaction tests have been shown to be more reliable for detecting Strongyloides compared with parasitological methods. Finally, larvae may be visualized by skin biopsy in patients with rash.

The appropriate treatment for strongyloidiasis is ivermectin with albendazole as an alternative. ${ }^{13}$

Ivermectin is administered as two single 200 $\mathrm{mcg} / \mathrm{kg}$ doses on two consecutive days, while albendazole $400 \mathrm{mg}$ as twice daily for three to seven days. Literature reports lower efficacy for albendazole than ivermectin. ${ }^{14}$

The prognosis of strongyloidiasis is usually good except in case of disseminated infection (hyperinfection syndrome), which could be lethal especially in people with weakened immune systems. ${ }^{15}$

The blurred clinical presentation in an immunocompetent patient, living at unusual latitudes for this type of parasite, delayed our diagnosis and made it complicated. Moreover, we observed a transformative multi-organic involvement: from a predominantly abdominal pattern with epigastric pain, cholestatic hepatitis with migrating stenosis of the main biliary duct, to a change within few days into a prevailing pulmonary involvement, with increasing dyspnea, polyserositis and eosinophilic pleural effusion.

Excluding other causes of eosinophilic pleural effusion (Table 2), it is important to keep in mind helminthic parasites.

Table 2. Some causes of eosinophilic pleural effusion and reasons for exclusion in our case.

\begin{tabular}{|c|c|}
\hline $\begin{array}{l}\text { Causes of eosinophil } \\
\text { pleural effusion }\end{array}$ & Reasons for exclusion of our case \\
\hline Pneumothorax & Excluded with computed tomography \\
\hline Hemothorax & Excluded with thoracentesis \\
\hline Pulmonary infarction & Excluded with computed tomography \\
\hline Several thoracenteses & Perform 2 thoracentesis only \\
\hline Neoplasia & $\begin{array}{l}\text { No evidence of solid or hematological } \\
\text { neoplasms }\end{array}$ \\
\hline Lymphoma & No evidence of lymphoma \\
\hline Tuberculosis & Quantiferon negative \\
\hline $\begin{array}{l}\text { Pneumotoxicity } \\
\text { (www.pneumotox.com) }\end{array}$ & $\begin{array}{l}\text { The patient does not take any medication } \\
\text { continuously }\end{array}$ \\
\hline Asbestosis & No evidence \\
\hline Parasitosis & $\begin{array}{l}\text { Strongyloides stercoralis positive } \\
\text { on stool sample }\end{array}$ \\
\hline Mycotic infection & $\begin{array}{l}\text { Negative laboratory serological } \\
\text { investigations }\end{array}$ \\
\hline Churg-Strauss syndrome & $\begin{array}{l}\text { Anti-neutrophil cytoplasmic antibody } \\
\text { negative }\end{array}$ \\
\hline
\end{tabular}


In conclusion, this wide range of clinical presentations, the non-specificity of laboratory parameters, make the diagnosis of strongyloidiasis definitely challenging for clinicians.

\section{References}

1. Adel AF. Mahmoud. Strongiloidiasis. Clin Inf Dis 1996;23:949-53.

2. Teixeira MC, Inês EJ, Pacheco FT, et al. Asymptomatic Strongyloides stercoralis hyperinfection in an alcoholic patient with intense anemia. J Parasitol 2010;96: 833-5.

3. Gulbas Z, Kebapci M, Pasaoglu O, Vardareli E. Successfull ivermectin treatment of hepatic strongiloidiasis preenting with severe eosinophilia. South Med J 2004;97:907-10.

4. Schär F, Trostdorf U, Giardina F, et al. Strongyloides stercoralis: global distribution and risk factors. PLoS Negl Trop Dis 2013;7:e2288.

5. Gyorkos TW, Genta RM, Viens P, MacLean JD. Seroepidemiology of Strongyloides infection in the Southeast Asian refugee population in Canada. Am J Epidemiol 1990;132:257-64.

6. Jourdan PM, Lamberton PHL, Fenwick A, Addiss DG. Soil-transmitted helminth infections. Lancet 2017 [Epub ahead of print].

7. Keiser PB, Nutman TB. Strongyloides stercoralis in the immunocompromised population. Clin Microbiol Rev 2004;17:208-17.

8. Newberry AM, Williams DN, Stauffer WM, et al. Strongyloides hyperinfection presenting as acute respiratory failure and gram-negative sepsis. Chest 2005; 128:3681-4.

9. Berkmen YM, Rabinowitz J. Gastrointestinal manifestations of the stongyloidiasis. Am J Roentgenol Radium Ther Nucl Med 1972;115:306-11.

10. Chu E, Whitlock WL, Dietrich RA. Pulmonary hyperinfection syndrome with Strongyloides stercoralis. Chest 1990;97:1475-7.

11. Mackey SL, Wagner KF. Dermatologic manifestations of parasitic diseases. Infect Dis Clin North Am 1994;8: 713-43.

12. Williams J, Nunley D, Dralle W, et al. Diagnosis of pulmonary strongyloidiasis by bronchoalveolar lavage. Chest 1988;94:643-4.

13. The Medical Letter, Inc. Drugs for parasitic infections, 3rd ed. New Rochelle, NY: The Medical Letter; 2013. Available from: https://www.uab.edu/medicine/gorgas /images/docs/syllabus/2015/03_Parasites/RxParasitesMe dicalLetter2013.pdf

14. Henriquez-Camacho C, Gotuzzo E, Echevarria J, et al. Ivermectin versus albendazole or thiabendazole for Strongyloides stercoralis infection. Cochrane Database Syst Rev 2016;(1):CD007745.

15. Longworth DL, Weller PF. Hyperinfection syndrome with strongyloidiasis. In: Remington JS, Swartz MN, eds. Current clinical topics in infectious diseases. New York, NY: McGraw-Hill; 1986. p.1. 